\title{
Risk factors for disease progression in HER2-positive breast cancer patients based on the location of metastases
}

\author{
Joanna Huszno, Elżbieta Nowara \\ Clinical and Experimental Oncology Department, Maria Skłodowska-Curie Memorial Cancer Center and Institute of Oncology, \\ Gliwice Branch, Gliwice, Poland
}

\begin{abstract}
Introduction: Trastuzumab therapy significantly improves progression-free and overall survival in HER2-positive [HER2(+)] breast cancer (BC) patients. However, in most patients with HER2(+) metastatic BC, the disease progress occurred. The aim of this study was to evaluate the clinicopathological risk factors for progression in HER2-positive breast cancer patients during trastuzumab therapy.

Material and methods: The analysis included medical records of HER2(+) metastatic BC patients treated with trastuzumab between 2006 and 2013.

Results: The most common site of progression during trastuzumab therapy were lungs 25 (39\%), central nervous system (CNS) 8 (13\%), skin 9 (14\%), locoregional lymph nodes $19(30 \%)$, liver $18(28 \%)$ and bone 17 (27\%). Patients with lung metastases significantly more often had a history of cancer in the family than women with other metastasis sites ( $24 \%$ vs. $2.6 \%$ ), $p=0.048$. Metastases to lungs occurred also more often during therapy containing trastuzumab with chemotherapy than trastuzumab alone $17 / 8$ (58\% vs. $41 \%$ ), $p=0.043$. Central nervous system metastases were observed insignificantly more frequently in postmenopausal women than premenopausal patients $8 / 0(22 \%$ vs. $0 \%), p=0.093$. There was reported a tendency to liver metastases in ER-negative tumors $13 / 20(72 \%$ vs. $44 \%, p=0.053)$. Bone metastases were associated with the positive steroid receptor status $(p=0.019)$ and second neoplasm in history $(p=0.06)$.

Conclusions: Risk factors for disease progression were the menopausal status (CNS metastases), steroid receptor status (liver, lymph nodes and bone metastases), history of cancer in the family (lung metastases) and history of cigarette smoking (liver metastases).
\end{abstract}

Key words: metastatic breast cancer, HER2 overexpression, trastuzumab, risk factors, metastasis location.

\section{Introduction}

The HER2/neu (ERBB2) oncogene is amplified and/or overexpressed in approximately $20 \%$ of breast cancers. It is known as a strong prognostic factor for relapse and poor overall survival, particularly in node-positive patients. Treatment with the anti-HER2 humanized monoclonal antibody, trastuzumab, significantly improves progression-free and overall survival among patients with HER2positive [HER2(+)] breast cancer (BC). However, in most patients with $\mathrm{HER} 2(+)$ metastatic $B C$, the disease progress occurred. In nearly half of HER2(+) BC patients, brain metastases may develop [1]. Therefore, HER2(+) is thought to be a risk factor for disease progression for metastatic BC patients. Only a few studies evaluated the correlation between clinicopathological features and development of distant metastases. Most of them concerned risk factors for brain metastases [2]. The authors did not find any papers presenting risk factors for disease progression due to metastasis location for HER2(+) BC patients.
The aim of this study was to evaluate the clinicopathological risk factors, such as patient age, menopausal status, comorbid conditions, overweight, history of cigarette smoking, history of neoplasms in the first degree family, the presence of the second neoplasm, steroid receptor status and the first site of metastases for progression in HER2(+) BC patients during trastuzumab therapy.

\section{Material and methods}

This retrospective study included medical records of 64 metastatic HER2(+) BC patients treated with trastuzumab between 2006 and 2013 in the Cancer Center and Institute of Oncology in Gliwice, Poland. The analysis of patients' medical records was performed according to national laws. All patients had BC confirmed with a microscopic examination. The study group comprised breast cancer patients with HER2 receptor overexpres- 


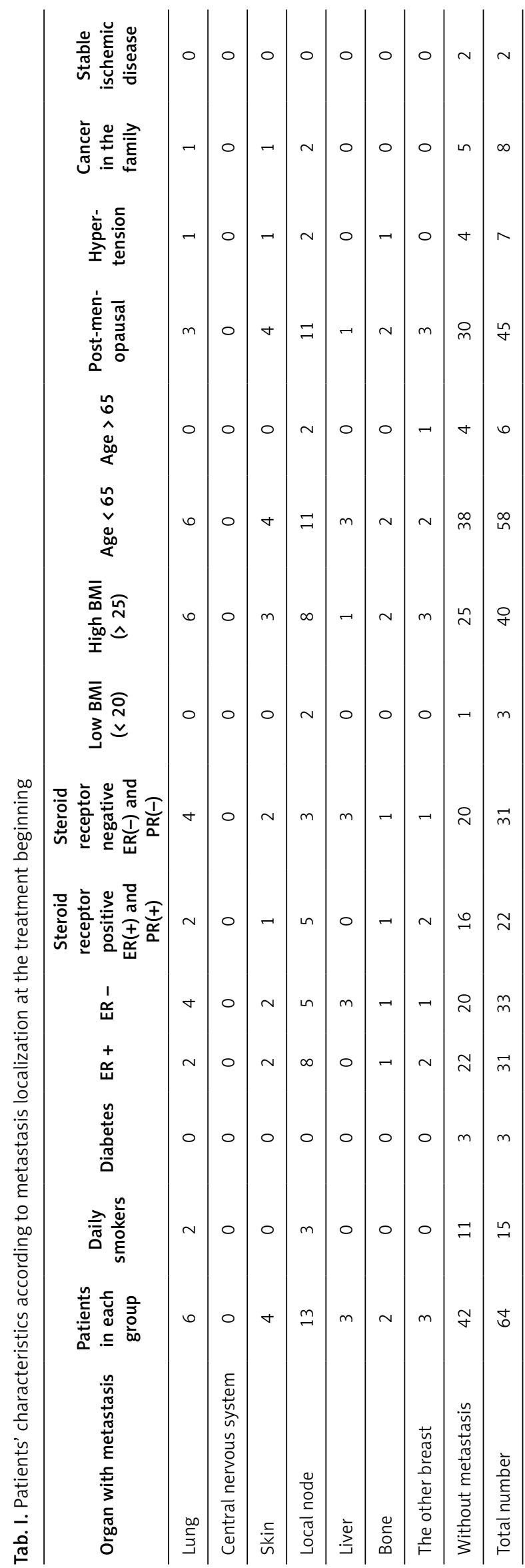

sion or HER2 gene amplification. HER2 overexpression was assessed using an immunohistochemical method (IHC) in postoperative specimens or samples obtained by a thick needle biopsy. HER2 gene amplification was additionally assessed by fluorescence in situ hybridization (FISH) in 8 patients. All patients were women. Inclusion criteria for trastuzumab therapy were baseline left ventricular ejection fraction (LVEF) $>50 \%$ and medical history without serious comorbid conditions such as unstable ischemic heart disease, valvular heart disease, chronic hypertension with cardiovascular problems or uncontrolled diabetes (uncontrolled diabetes was classified as a random blood glucose level $\geq 11.0 \mathrm{mmol} / \mathrm{l}$ in diabetic patients). Subsequent inclusion criteria were age above 18, performance status ZUBROD 0-1, the peripheral blood count, renal and liver function within normal values and complete medical documentation including course of disease and follow-up.

The data, including the age at onset, menopausal status, comorbid conditions, overweight, the history of cigarette smoking, history of neoplasms in the first degree family, the presence of the second neoplasm, disease stage according to TNM classification, surgical procedures, histology, estrogen and progesterone receptor status, HER2 status and contralateral breast cancer were gathered from hospital records and pathology reports.

Echocardiography was performed every 3 infusions during trastuzumab therapy and also before and after the anthracycline-based chemotherapy. Cardiac side effects were assessed in the NYHA (New York Heart Association) classification and other toxicity in the CTCAE scale (ver. 4.0). The earliest manifestations of myocardial damage diagnosed by echocardiography were LVEF decrease, abnormalities of the right ventricular contractility, ventricular dilation and abnormalities of the left ventricular contractility. Treatment response was assessed due to RECIST 1.0.

Statistical analysis was carried out using STATISTICA 7 software. The frequency of side effects was calculated. The qualitative features were presented as a percentage of their occurrence and evaluated with Fisher's test and $\chi^{2}$ test with Yates' correction. Differences were considered as significant if the $p$ value was $<0.05$. Confounding and effect-modifying variables were studied by multivariate analysis.

\section{Results}

The analysis included 64 metastatic BC patients treated with trastuzumab. The median age at diagnosis was 52 years (range 27-70). Patients' characteristics according to metastasis localization at the treatment beginning are shown in Table I. Thirty-three patients (52\%) received trastuzumab in combination with chemotherapy and the remaining 31 (48\%) in monotherapy. The most frequently used cytostatics were paclitaxel or 
vinorelbine. The median number of trastuzumab infusions was 19 (range 2-118). The duration of trastuzumab treatment ranged from 2 months to 7 years (mean 14 months). Almost one fifth of patients was treated for more than 3 years.

Disease progression during trastuzumab therapy occurred in 49 (76\%) patients. Treatment discontinuation due to progression in the form of the appearance of new metastases was observed in 24 (49\%) of them. The remaining women (40\%) completed therapy due to progression in the form of enlargement of the lesions which were present at the beginning of the treatment. The information on metastasis localization at the treatment start is presented in Table II. Disease stagnation was observed in 9 (14\%) patients. The best trastuzum$a b$ therapy response $(C R+P R)$ was observed in $10 \%$ of women, especially with metastases to the skin, lungs and locoregional lymph nodes.

The most common sites of progression during trastuzumab therapy were lungs 25 (39\%), central nervous system (CNS) 8 (13\%), skin 9 (14\%), locoregional lymph nodes 19 (30\%), liver 18 (28\%) and bone 17 (27\%). The patients with lung metastases were non-significantly younger than women with other metastasis locations ( $<65$ years) $24 / 34$ ( $96 \%$ vs. $87 \%$ ), $p=0.390$. There was no association between lung metastases and the premenopausal status $8 / 11$ (32\% vs. $28 \%, p=0.784)$, obesity (BMI > 25) 15/25 (60\% vs. 64\%, $p=0.784)$, hypertension $2 / 5$ ( $8 \%$ vs. $13 \%, p=0.695)$ or diabetes $1 / 2$ ( $4 \%$ vs. $5 \%$ ). There were also no differences between the patients with lung metastases with other metastasis sites according to the negative steroid receptor status $14 / 17$ ( $56 \%$ vs. $44 \%), p=0.442$. Patients with lung metastases significantly more often had a history of cancer in the family than women with other metastasis sites ( $24 \%$ vs. $2.6 \%), p=0.048$. Disease dissemination to lungs was detected more frequently in the patients without metastases at the baseline compared to the patients with other metastasis sites at baseline (Table II). Metastases to lungs occurred also more often during therapy containing trastuzumab with chemotherapy than trastuzumab alone $17 / 8$ (52\% vs. $41 \%), p=0.043$.

Central nervous system metastases were observed insignificantly more frequently in postmenopausal women than premenopausal patients $8 / 0$ (22\% vs. $0 \%), p=0.093$. There was no association between overweight $5 / 35$ (63\% vs. $63 \%$ ), diabetes $0 / 5$ (0 vs. $5 \%$ ), hypertension $1 / 6(13 \%$ vs. $11 \%)$ or cardiovascular disease $0 / 2$ (0\% vs. $4 \%$ ) and CNS metastases. Dissemination to CNS was insignificantly more often observed in the patients with the negative steroid receptor status 5/26 (63\% vs. $46 \%), p=0.468$. Most patients with CNS metastases (63\%) were without metastases at baseline. In the multivariate analysis, postmenopausal BC patients with liver as the first site of metastasis were more prone to develop CNS metastases during trastuzumab therapy $(p=0.178)$.

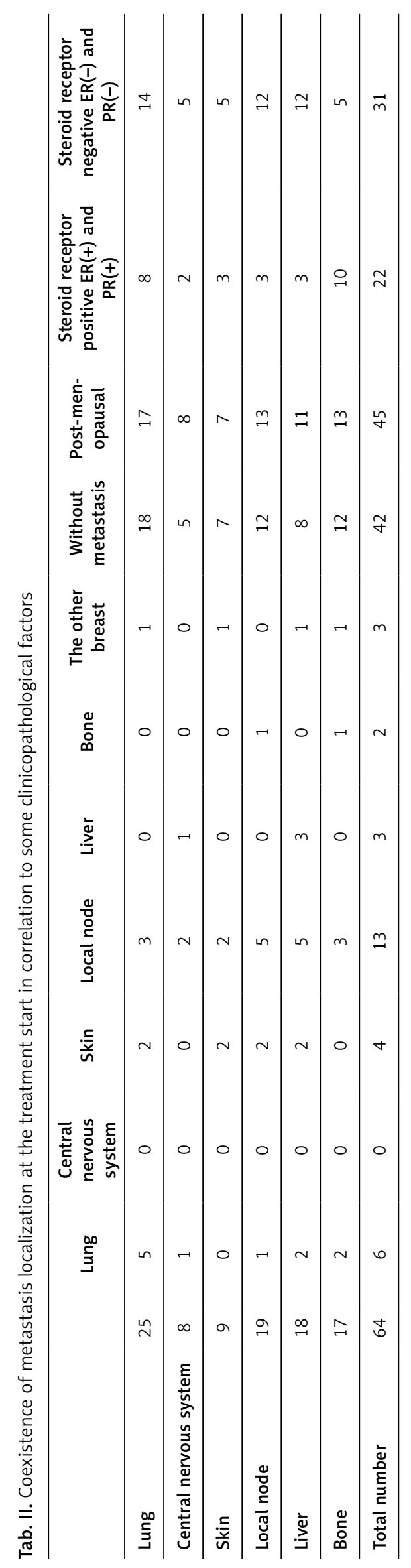


There was observed a tendency to the presence of liver metastases in patients without any history of breast cancer in the family in comparison with other metastasis location $39 / 17$ ( $15 \%$ vs. $6 \%, p=0.424$ ). Women with liver metastases had also less frequently the second neoplasm in history $1 / 17$ (13\% vs. 33\%, $p=$ $0.225)$. There were reported no differences according to the age (<65 years) $1 / 5$ ( $6 \%$ vs. $11 \%, p=0.667$ ), menopausal status (premenopausal) $7 / 12$ (39\% vs. $26 \%$ ), $p=0.368$, overweight $(\mathrm{BMI}>25) 10 / 30$ (56\% vs. $65 \%$ ), $p=0.569$ and co-morbid conditions such as hypertension $2 / 5$ (11\% vs. $11 \%$ ), between patients with liver and other metastasis sites. None of the patients with liver metastases suffer from diabetes. A history of cigarette smoking was reported significantly more often in patients with locations other than liver 14/1 (30\% vs. $6 \%, p=0.048)$. There was observed a tendency to liver metastases in negative steroid receptor status tumors $13 / 20$ ( $72 \%$ vs. $44 \%, p=0.053)$. Liver metastases were observed with a similar frequency regardless of the type of treatment (trastuzumab alone vs. combination chemotherapy) $7 / 11$ ( $23 \%$ vs. $33 \%, p=0.410)$.

Locoregional lymph node metastases occurred insignificantly more often in patients with overweight $(\mathrm{BMI}>25) 15 / 25$ (79\% vs. 56\%) $p=0.096$ and in older women (> 65 years) ( $16 \%$ vs. $7 \%, p=0.351)$. The negative steroid receptor status was detected more frequently in patients with lymph node metastases in comparison to other metastasis sites $16 / 26$ (84\% vs. $58 \%, p=0.049)$. There were reported no differences according to the menopausal status (premenopausal status) $6 / 13$ (32\% vs. $29 \%$ ) and co-morbid conditions such as diabetes $2 / 1$ ( $11 \%$ vs. $2 \%, p=0.208)$ or hypertension $1 / 6$ ( $5 \%$ vs. $13 \%, p=0.664)$. There was no association between lymph node metastases and treatment type (trastuzumab alone vs. combination therapy) 9/10 (29\% vs. $30 \%)$.

Bone metastases were observed significantly more frequently in older (> 65 years) women than in younger patients $3 / 3(18 \%$ vs. $6 \%, p=0.329)$. Patients with bone metastases had significantly more often a positive steroid receptor status 10/12 (59\% vs. $26 \%$ ), $p=0.019$. There were reported no differences according to the menopausal status (premenopausal status) 4/15 (24\% vs. $32 \%, p=0.757$ ) and co-morbid conditions such as diabetes $1 / 2$ (6\% vs. $4 \%$ ) or hypertension $3 / 4$ ( $18 \%$ vs. $9 \%, p=0.370)$. There was a tendency to bone dissemination in patients who received trastuzumab alone in comparison with combination therapy $11 / 6$ (65\% vs. $35 \%), p=0.159$.

The presence of skin metastases during trastuzumab therapy occurred nonsignificantly more often in patients above 65 years $2 / 6(20 \%$ vs. $13 \%)$ and overweight $7 / 2$ (20\% vs. $8 \%$ ), $p=0.332, p=0.052$, respectively. None of the patients with skin metastases had history of breast cancer in the family $0 / 7$ ( 0 vs. $13 \%$ ). There was no association between skin metastases and treatment type (trastuzumab alone vs. combination therapy) 5/4 (31\% vs. $33 \%$ ), $p=0.729$.

\section{Discussion}

In the present study we consider whether depending on metastatic sites, the presence of clinicopathological features such as the menopausal status, steroid receptor status and history of cancer in the family may be predictive factors for disease progression.

The most papers concern risk factors for brain relapse in metastatic breast cancer patients. Metastases to CNS occur in $10-16 \%$ of patients but in autopsy examinations, the brain metastases are found in about $30 \%$ of patients $[3,4]$. Although trastuzumab improves survival in metastatic breast cancer patients with HER2 overexpression, $40 \%$ of them develop brain metastasis [1]. In some studies, CNS metastases were found more frequently in younger, premenopausal patients and those with a negative steroid receptor status [5]. Other analyses have shown an association between the tumor size, grade, nodal status, HER2 status and CNS metastases [6]. The type of adjuvant treatment did not influence CNS recurrences [5, 7]. Only a few studies evaluated the association between the first sites of metastasis and further development to CNS. Most of them reported that the presence of lung metastases was strongly associated with the occurrence of brain metastases. Slimane et al. have shown that the presence of lung metastases and negative hormone receptor status are predictive factors for the occurrence of brain metastases [8, 9]. In another study, patients with CNS metastases were more likely to have bone metastases ( $p<0.013$ ) [10]. Altudag et al. described lung (20\%), bone (18\%) and then liver (6\%) as the most common site of metastasis before CNS dissemination. Favorable prognostic factors in this group were younger age and ER status [11]. In a study which described the results of autopsies performed on women who died of $\mathrm{BC}$, the most common site of metastases was lungs/ pleura (80\%), bone (74\%), liver (71\%) and then non axillary lymph nodes (55\%). Patients with CNS dissemination more often had bone metastases $[12,13]$. In the present paper, CNS dissemination during trastuzumab therapy was found more frequently in postmenopausal patients but under 65 years and with a negative steroid receptor status. Patients without metastases at baseline more often develop brain metastases. Any location of metastases did not influence CNS dissemination.

In a trial conducted by Cummings et al., younger age - under 49 years $(p<0.001)$ and surgical excision of the primary tumor $(p=0.002)$ were associated with metastasis to the liver [12]. History of breast cancer in the family, history of cigarette smoking and a negative steroid receptor status were found in our study to be 
a predictive factor for liver metastases during trastuzumab therapy.

In the analyzed group of patients, the most frequent location of progression during trastuzumab therapy was lungs. Disease dissemination to lungs was detected more frequently in the patients without metastases at baseline compared to patients with other metastases sites at baseline. Other risk factors were age under 65 years and a history of cancer in the family. The remaining clinicopathological factors did not influence the development of lung dissemination in our analysis. Previous studies described only the association between lungs as the first site of metastasis and CNS dissemination [11-13]. As for now, there are no data evaluating risk factors for lung dissemination during trastuzumab therapy.

Bones are the most common site for metastatic dissemination in BC patients [14]. Some authors described the highest bone metastasis incidence in a group with four or more axillary lymph nodes dissemination and in the first local recurrence, in soft tissue or locoregional lymph nodes. No association between the menopausal status and bone metastases was presented. Patients with ER negative tumors or tumors with low ER expression had a higher rate of bone metastases than patients with a positive steroid receptor status. However, ER positive breast cancer may be predictive factor for a higher incidence of bone metastases [15]. The analysis of histological factors did not confirm the role of age, menopausal status, the number of regional lymph node metastases, tumor size, mitotic counts nuclear atypia, fat and vascular invasion as risk factors for bone metastases [16]. We found that older patients had a higher incidence of bone metastases. The other risk factor for bone metastases was the steroid receptor status. The association between metastasis location at diagnosis and bone dissemination was not presented.

In some studies, trastuzumab was continued after disease progression due to a potential synergistic interaction with second-line chemotherapy [17]. Cancello et al. described a statistically insignificant trend to improve survival for patients receiving trastuzumab with multiple lines of systemic treatment [18]. In the studied group, patients with disease progression during trastuzumab therapy did not continue therapy due to the reimbursement system. Usually they received capecitabine with lapatinib.

\section{Conclusions}

The best response to trastuzumab therapy was observed in the patients with metastases to the skin, lungs and locoregional lymph nodes. The most frequent location of progression during trastuzumab therapy was lungs and lymph nodes. Risk factors for disease progression were the menopausal status (CNS metastases), steroid receptor status (liver, lymph nodes and bone metastases), history of cancer in the family (lung metastases) and history of cigarette smoking (liver metastases).

\section{Disclosure}

Authors report no conflict of interest.

\section{References}

1. Park IH, Ro J, Lee KS, et al. Trastuzmab treatment beyond brain progression in HER2 positive metastatic breast cancer. Ann Oncol 2009; 20: 56-62.

2. Pestalozzi BC. Brain metastases and subtypes of breast cancer. Ann Oncol 2009; 20: 803-805.

3. Lin NU, Bellon JR, Winter EP. CNS metastases in breast cancer. J Clin Oncol 2004; 22: 3608-3617.

4. Weil RJ, Palmieri DC, Bronder JL, et al. Breast cancer metastasis to the central nervous system. Am J Pathol 2005; 167: 913-920.

5. Pestalozzi BC, Francis $P$, Quinaux $E$, et al. Is risk of central nervous system (CNS) relapse related to adjuvant taxane treatment in node-positive breast cancer? Results of the CNS substudy in the intergroup phase III BIG 02-98 trial. Ann Oncol 2008; 19: 1837-1841.

6. Pestalozzi BC, Zahrieh D, Price KN, et al. Identifying breast cancer patients at risk for central nervous system (CNS) metastases in trials of the International Breast Cancer Study Group (IBCSG). Ann Oncol 2006; 17: 935-944.

7. Tham YL, Sexton K, Kramer R, et al. Primary breast cancer phenotypes associated with propensity for central nervous system metastases. Cancer 2006; 107: 696-704

8. Slimane K, Andre F, Delaloge A, et al. Risk factors for brain relapse in patients with metastatic breast cancer. Ann Oncol 2004; 15: 1640-1644.

9. Crivellari D, Pagani O, Veronesi A, et al. High incidence of central nervous system involvement inpatients with metastatic or locally advanced breast cancer treated with epirubicin and docetaxel. Ann Oncol 2001; 12: 353-356.

10. Kennecke H, Yerushalmi R, Woods R, et al. Metastatic Behavior of Breast Cancer Subtypes. J Clin Oncol 2010; 28: 3271-3277.

11. Altundag K, Bondy ML, Mirza NQ, et al. Clinicopathologic characteristics and prognostic factors in 420 metastatic breast cancer patients with central nervous system metastasis. Cancer 2007; 110: 2640-2647.

12. Cummings MC, Simpson PT, Reid LE, et al. Metastatic progression of breast cancer: insights from 50 years of autopsies. J Pathol 2014; 232: 23-31.

13. Slimane K, Andrae F, Delaloge $S$, et al. Risk factors for brain relapse in patients with metastatic breast cancer. Ann Oncol 2004; 15: 1640-1644.

14. Coleman RE. Clinical features of metastatic bone disease and risk of skeletal morbidity. Clin Cancer Res 2006; 12: 6243-6249.

15. Colleoni M, O'Neill A, Goldhirsch A, et al. Identifying Breast Cancer Patients at High Risk for Bone Metastases. J Clin Oncol 2000; 18: 3925-3935.

16. Koyama T, Hasebe T, Tsuda $\mathrm{H}$, et al. Histological factors associated with initial bone metastasis of invasive ductal carcinoma of the breast. Jpn J Cancer Res 1999; 90: 294-300.

17. Bartsch R, Wenzel C, Hussian D, et al. Analysis of trastuzumab and chemotherapy in advanced breast cancer after the failure of at least one earlier combination: an observational study. BMC Cancer 2006; 6: 63.

18. Cancello G, Montagna E, D’Agostino D, et al. Continuing trastuzumab beyond disease progression: outcomes analysis in patients with metastatic breast cancer. Breast Cancer Res 2008; 10: R60. 